\title{
GENERALIZATIONS OF COPSON'S INEQUALITITS INVOLVING SERIES OF POSITIVE TERMS
}

\author{
DAH-YAN HWANG
}

\begin{abstract}
The aim of the present paper is to establish some new inequalities involving series of positive terms.
\end{abstract}

\section{Introduction}

In [1] Copson established the following Hardy's inequalities [4, Theorem 326 and Theorem 331] involving series of positive terms.

Theorem A. If $p>1, \lambda_{n}>0, a_{n}>0, \Lambda_{n}=\sum_{i=1}^{n} \lambda_{i}, A_{n}=\sum_{i=1}^{n} \lambda_{i} a_{i}$ and $\sum_{n=1}^{\infty} \lambda_{n} a_{n}^{p}$ converges, then

$$
\sum_{n=1}^{\infty} \lambda_{n}\left(\frac{A_{n}}{\Lambda_{n}}\right)^{\dot{p}} \leq\left(\frac{p}{p-1}\right)^{p} \sum_{n=1}^{\infty} \lambda_{n} a_{n}^{p} .
$$

The constant is the best possible.

Theorem B. Let $p, \lambda_{n}, a_{n}, \Lambda_{n}$ be defined as in Theorem $A$. If $A_{n}=\sum_{i=n}^{\infty} \frac{\lambda_{j} a_{i}}{\Lambda_{i}}$ and $\sum_{n=1}^{\infty} \lambda_{n} a_{n}^{p}$ converges, then

$$
\sum_{n=1}^{\infty} \lambda_{n} A_{n}^{p} \leq p^{p} \sum_{n=1}^{\infty} \lambda_{n} a_{n}^{p}
$$

The constant is the best possible.

There is a vast literature which deals with alternative proofs, generalizations and extensions of (1) and (2), see $[2,3,5,6,8,12]$ and the references given therein. In the present paper we establish some new inequalities involving series of positive terms which claim their origin to the inequalities given in (1) and (2).

Received October 21, 1996.

1991 Mathematics Subject Classification. Primary 26D15. Key words and phrases. Hardy's inequality, Copson's inequality, Jensen's inequality Hölder's
inequality, Young's inequality. 


\section{Main results}

The following Theorems are base on the idea used by Levinson [7] to obtain the interesting generalizations of Hardy's integral inequality and Pachpattc and Love [1.1] to obtain inequalities related to Hardy's integral inequality. In this section, we establish some discrete analogue of theirs. Here we assume that the left, sides of inequalities exist when right sides do.

Theorem 1. For $n=1,2,3, \ldots$ and $m=1,2,3, \ldots, M$, let $p>1, a(n)>0, \lambda_{m}(n)>$ $0, \beta_{m}(n)>0, \Lambda_{m}(n)=\sum_{i=1}^{n} \lambda_{m}(i) \beta_{m}(i), I_{m} a(n)=\frac{1}{\Lambda_{m}(n)} \sum_{i=1}^{n} \lambda_{m}(i) \beta_{m}(i) a(i), \Lambda_{m}(n)$ $=I_{m} I_{m-1} \cdots I_{1} a(n), A_{0}(n)=a(n)$, where $M$ is a positive integer, and further let $\sum_{n=1}^{\infty} \lambda_{1}(n) A_{0}^{\Gamma}(n)$ converge. If there exists $k_{m} \geq p /(p-1)$ such that

$$
p-1+\frac{\left[\beta_{m}(n+1)-\beta_{m}(n)\right] \Lambda_{m}(n)}{\beta_{m}(n+1) \beta_{m}(n) \lambda_{m}(n)} \geq \frac{p}{k_{m}}, \text { and } \lambda_{1}(n) \geq \lambda_{2}(n) \geq \cdots \geq \lambda_{M}(n),
$$

then

$$
\sum_{n=1}^{\infty} \lambda_{M}(n) A_{M}^{p}(n) \leq\left(\pi_{m=1}^{M} k_{m}\right)^{p} \sum_{n=1}^{\infty} \lambda_{1}(n) A_{0}^{p}(n)
$$

the constant in (4) is the best possible.

Proof. Let $\lambda_{m}(0)=\beta_{m}(0)=1$ and agree that $\Lambda_{m}(0)=0$ for $m=1,2,3, \ldots, M$. For $n=0,1,2, \ldots$ and $m=1,2,3, \ldots, M$,

$$
\begin{aligned}
& -p \lambda_{m}(n+1) A_{m-1}(n+1) A_{m}^{p-1}(n+1) \\
= & -p \lambda_{m}(n+1) \beta_{m}(n+1) A_{m-1}(n+1)\left[A_{m}^{p-1}(n+1) / \beta_{m}(n+1)\right] \\
= & -p\left[A_{m}(n+1) \Lambda_{m}(n+1)-A_{m}(n) \Lambda_{m}(n)\right]\left[A_{m}^{p-1}(n+1) / \beta_{m}(n+1)\right] \\
= & -p A_{m}^{p}(n+1)\left[\Lambda_{m}(n+1) / \beta_{m}(n+1)\right]+p\left[\Lambda_{m}(n) / \beta_{m}(n+1)\right] \Lambda_{m}(n) A_{m}^{p-1}(n+1) \\
\leq & -p A_{m}^{p}(n+1)\left[\Lambda_{m}(n+1) / \beta_{m}(n+1)\right]+\left[\Lambda_{m}(n) / \beta_{m}(n+1)\right] \cdot A_{m}^{n}(n) \\
& \left.+(p-1)\left[\Lambda_{m}(n) / \beta_{m}(n+1)\right]\right] A_{m}^{p}(n+1),
\end{aligned}
$$

the lasi inequality follows from Young's inequality i.c.

$k x y^{k-1} \leq x^{k}+(k-1) y^{k}, x, y \geq 0, k>1$.

Hence

$$
\begin{aligned}
& (p-1) \lambda_{m}(n+1) A_{m}^{p}(n+1)+\frac{\left[\beta_{m}(n+1)-\beta_{m}(n)\right] \Lambda_{m}(n)}{\beta_{m}(n+1) \beta_{m}(n) \lambda_{m}(n)} \lambda_{m}(n) A_{m}^{p}(n) \\
& -p \lambda_{m}(n+1) A_{m-1}(n+1) A_{m}^{p-1}(n+1) \\
\leq & (p-1) \lambda_{m}(n+1) A_{m}^{p}(n+1)+\frac{\left[\beta_{m}(n+1)-\beta_{m}(n)\right] \Lambda_{m}(n)}{\beta_{m}(n+1) \beta_{m}(n)} A_{m}^{p}(n) \\
& -p A_{m}^{n}(n+1)\left[\Lambda_{m}(n+1) / \beta_{m}(n+1)\right] \\
& +\left[\Lambda_{m}(n) / \beta_{m}(n+1)\right] A_{m}^{p}(n)+(p-1)\left[\Lambda_{m}(n) / \beta(n+1)\right] A_{m}^{p}(n+1) \\
= & \frac{\Lambda_{m}(n) A_{m}^{p}(n)}{\beta_{m}(n)}-\frac{\Lambda_{m}(n+1) A_{m}^{p}(n+1)}{\beta_{m}(n+1)}
\end{aligned}
$$


By adding the inequalities for $n=0,1,2, \ldots, N-1$, we have

$$
\begin{aligned}
& \sum_{n=0}^{N-1}(p-1) \lambda_{m}(n+1) A_{m}^{p}(n+1)+\sum_{n=0}^{N-1} \frac{\left[\beta_{m}(n+1)-\beta_{m}(n)\right] \Lambda_{m}(n)}{\beta_{m}(n+1) \beta_{m}(n) \lambda_{m}(n)} \lambda_{m}(n) A_{m}^{p}(n) \\
& -p \sum_{n=0}^{N-1} \lambda_{m}(n+1) A_{m-1}(n+1) A_{m}^{p-1}(n+1) \\
\leq & -\left[\Lambda(N) / \beta_{m}(N)\right] A_{m}^{p}(N) \leq 0 .
\end{aligned}
$$

Thus,

$$
\begin{aligned}
& \sum_{n=1}^{N}(p-1) \lambda_{m}(n) A_{m}^{p}(n)+\sum_{n=1}^{N-1} \frac{\left[\beta_{m}(n+1)-\beta_{m}(n)\right] \Lambda_{m}(n)}{\beta_{m}(n+1) \beta_{m}(n) \lambda_{m}(n)} \lambda_{m}(n) A_{m}^{p}(n) \\
\leq & p \sum_{n=1}^{N} \lambda_{m}(n) A_{m-1}(n) A_{m}^{p-1}(n) .
\end{aligned}
$$

Using (3) and the assumption that $k_{m} \geq p /(p-1)$, we have

$$
\sum_{n=1}^{N} \lambda_{m}(n) A_{m}^{p}(n) \leq K_{m} \sum_{n=1}^{N} \lambda_{m}(n) A_{m-1}(n) A_{m}^{p-1}(n)
$$

Using Hölder inequality with indices $p$ and $p /(p-1)$, we have

$$
\begin{aligned}
\sum_{n=1}^{N} \lambda_{m}(n) A_{m-1}(n) A_{m}^{p-1}(n) & =\sum_{n=1}^{N} \lambda_{m}^{1 / p}(n) A_{m-1}(n) \lambda_{m}^{(p-1) / p}(n) A_{m}^{p-1}(n) \\
& \leq\left[\sum_{n=1}^{N} \lambda_{m}(n) A_{m-1}^{p}(n)\right]^{1 / p}\left[\sum_{n=1}^{N} \lambda_{m}(n) A_{m}^{p}(n)\right]^{(p-1) / p}
\end{aligned}
$$

which together with (5), imply

$$
\sum_{n=1}^{N} \lambda_{m}(n) A_{m}^{p}(n) \leq k_{m}\left[\sum_{n=1}^{N} \lambda_{m}(n) A_{m-1}^{p}(n)\right]^{1 / p}\left[\sum_{n=1}^{N} \lambda_{m}(n) A_{m}^{p}(n)\right]^{(p-1) / p}
$$

Dividing the above inequality by the last factor on the right side and raising the result to the pth power, we obtain

$$
\sum_{n=1}^{N} \lambda_{m}(n) A_{m}^{p}(n) \leq k_{m}^{p} \sum_{n=1}^{N} \lambda_{m}(n) A_{m-1}^{p}(n) .
$$

Now since $\lambda_{1}(n) \geq \lambda_{2}(n) \geq \cdots \geq \lambda_{M}(n)$ for $n=1,2,3, \ldots$, we have

$$
\sum_{n=1}^{N} \lambda_{M}(n) A_{M}^{P}(n) \leq\left(\pi_{m=1}^{M} K_{m}\right)^{p} \sum_{n=1}^{N} \lambda_{1}(n) A_{0}^{p}(n) .
$$


The desired incquality (4) then follows from (6) by letting $N$ tend to infinity.

The case $M=1, \beta_{1}(n)=1, n=1,2,3, \ldots, k_{1}=p /(p-1)$ show constant in (4) is the best possible.

Remark 1 . Theorem 1 redues to Theorem 1 in [5] when $M=1$ and reduces io (1) when $M=1, K_{1}=p /(p-1)$ and $\beta_{1}(n)=1$ for $n=1,2,3, \ldots$

Theorem 2. Let $H$ be a real-valued positive convex function defincd on $(0, \infty)$, and let $p, a(n), \lambda_{m}(n), \beta_{m}(n), \Lambda_{m}(n), I_{m} a(n), A_{m}(n), A_{0}(n)$ and $k_{m}$ be as in Theorem 1 . If $\sum_{n=1}^{\infty} \lambda_{1}(n) H^{p}\left(A_{0}(n)\right)$ converges, then

$$
\sum_{n=1}^{\infty} \lambda_{M}(n) H^{p}\left(A_{M}(n)\right) \leq\left(\pi_{m=1}^{M} k_{m}\right)^{p} \sum_{n=1}^{\infty} \lambda_{1}(n) H^{p}\left(A_{0}(n)\right) .
$$

Proof. Since $H$ is a convex function, by repeated application of Jenscn's inequality; we obtain

$$
H\left(A_{M}(n)\right) \leq F(n), \quad \text { where } F(n)=I_{M} I_{M-1} \cdots I_{1} H(a(n)) .
$$

Thus

$$
\sum_{n=1}^{\infty} \lambda_{M}(n) H^{p}\left(\dot{A}_{M}(n)\right) \leq \sum_{n=1}^{\infty} \lambda_{M}(n) F^{p}(n) .
$$

Replace $a(n)$ by $H(a(n))$ is (4), we have

$$
\sum_{n=1}^{\infty} \lambda_{M}(n) F^{p}(n) \leq\left(\pi_{m=1}^{M} k_{m}\right)^{p} \sum_{n=1}^{\infty} \lambda_{1}(n) H^{p}(a(n)) .
$$

The inequality (7) then follows from (8) and (9).

Remark 2. The inequality (1) is the special case of the inequality $(7)$ when $H(u)=u$. Theorem 2 redues to Theorem 2 in [5] when $M=1$ and reduced to Theorem 1 in [12] when $M=1, k_{1}=p /(p-1)$ and $\beta_{1}(n)=1$, for $n=1,2,3, \ldots$ We note that the lasi case shows the constant in (7) is the best possible.

Theorem 3. Let $p, a(n), \lambda_{m}(n), \beta_{m}(n), \Lambda_{m}(n), I_{m} a(n), A_{m}(n), A_{0}(n)$ and $k_{m}$ be as in Theorem 1, and let $\varphi>0$ be defincd on $(0, \infty)$ so that $\varphi^{\prime \prime} \geq 0$ and

$$
\varphi \varphi^{\prime \prime} \geq(1-1 / p)\left(\varphi^{\prime}\right)^{2}
$$

If $\sum_{n=1}^{\infty} \lambda_{1}(n) \varphi\left(A_{0}(n)\right)$ converges, then

$$
\sum_{n=1}^{\infty} \lambda_{M}(n) \varphi\left(A_{M}(n)\right) \leq\left(\pi_{m=1}^{M} k_{m}\right)^{p} \sum_{n=1}^{\infty} \lambda_{1}(n) \varphi\left(A_{0}(n)\right)
$$


Proof. Let $\Psi(u)=\varphi^{1 / p}(u), u>0$. Then, by $(10), \psi^{\prime \prime} \geq 0$. Hence $\psi$ is convex on $(0, \infty)$. Thus, by Theorem 2 , we have

$$
\sum_{n=1}^{\infty} \lambda_{M}(n) \psi^{p}\left(A_{M}(n)\right) \leq\left(\pi_{m=1}^{M} k_{m}\right)^{p} \sum_{n=1}^{\infty} \lambda_{1}(n) \psi^{p}\left(A_{0}(n)\right)
$$

and therefore

$$
\sum_{n=1}^{\infty} \lambda_{M} \varphi\left(A_{M}(n)\right) \leq\left(\pi_{n=1}^{M} k_{m}\right)^{p} \sum_{n=1}^{\infty} \lambda_{1}(n) \varphi\left(A_{0}(n)\right) .
$$

This is the desired inequality (11).

Remark 3. Theorem 3 reduces to Theorem 2 and Theorem 1 when $\varphi(u)=H^{p}(u)$ and $\varphi(u)=u^{p}$, respectively. Theorem 3 reduces to Theorem 3 in [5] when $M=1$, and reduces to Theorem 1 in [12] when $M=1, \varphi(u)=H^{p}(u), k_{1}=p /(p-1)$ and $\beta_{1}(n)=1$, for $n=1,2, \ldots$. Also we note that the inequality (1) is the special case of the inequality (11) when $M=1, \varphi(u)=u^{p}, k_{1}=p /(p-1)$ and $\beta_{1}(n)=1$, for $n=1,2, \ldots$

Theorem 4. For $n=1,2,3, \ldots$ and $m=1,2, \ldots, M$ let $p>1, a(n)>0, \lambda_{m}(n-$ 1) $>0,\left\langle\beta_{m}(n-1)\right\rangle$ be non-increasing positive sequence, $\Lambda_{m}(n)=\sum_{i=1}^{n} \lambda_{m}(i) \beta_{m}(i)$, $\Lambda_{m}(0)=0, J_{m} a(n)=\sum_{i=n}^{\infty} \frac{\lambda_{m}(i) \beta_{m}(i) a(i)}{\Lambda_{m}(i)}, B_{m}(n)=J_{m} J_{m-1} \cdots J_{1} a(n), B_{0}(n)=a(n)$ and futher let $\sum_{n=1}^{\infty} \lambda_{m}(n) B_{m-1}^{p}(n)$ converge for each $m$. If there exist $k_{m} \geq p$ such that

$$
1-\frac{\left[\beta_{m}(n)-\beta_{m}(n-1)\right] \Lambda_{m}(n-1)}{\beta_{m}(n) \beta_{m}(n-1) \lambda_{m}(n)} \geq \frac{p}{k_{m}}
$$

and further if $\lambda_{1}(n) \geq \lambda_{2}(n) \geq \cdots \geq \lambda_{M}(n)$ then

$$
\sum_{n=1}^{\infty} \lambda_{M}(n) B_{M}^{p}(n) \leq\left(\pi_{m=1}^{M} k_{m}\right)^{p} \sum_{n=1}^{\infty} \lambda_{1}(n) B_{0}^{p}(n) .
$$

Proof. For $n=1,2, \ldots$ and $m=1,2, \ldots, M$, we have, by Hölder's inequality,

$$
\begin{aligned}
B_{m}^{p}(n) & =\left[\sum_{i=n}^{\infty} \lambda_{m}^{1 / p}(i) B_{m-1}(i) \lambda_{m}^{(p-1) / p}(i) \beta_{m}(i) \Lambda_{m}^{-1}(i)\right]^{p} \\
& \leq\left[\sum_{i=n}^{\infty} \lambda_{m}(i) B_{m-1}^{p}(i)\right]\left[\sum_{i=n}^{\infty} \lambda_{m}(i)\left(\frac{\Lambda_{m}(i)}{\beta_{m}(i)}\right)^{-p /(p-1)}\right]^{p-1} .
\end{aligned}
$$

Since

$$
\begin{aligned}
\sum_{i=n}^{\infty} \lambda_{m}(i)\left(\frac{\Lambda_{m}(i)}{\beta_{n}(i)}\right)^{-p /(p-1)} \leq & \sum_{i=n}^{\infty}\left[\lambda_{m}(i)+\frac{\left(\beta_{m}(i-1)-\beta_{m}(i)\right) \Lambda_{m}(i-1)}{\beta_{m}(i-1) \beta_{m}(i)}\right] \\
& \cdot\left(\frac{\Lambda_{m}(i)}{\beta_{m}(i)}\right)^{-p /(p-1)}
\end{aligned}
$$




$$
\begin{aligned}
& <\int_{\frac{\Lambda_{m}(n-1)}{\hat{\beta}_{m}(n-1)}}^{\infty} x^{-p /(p-1)} d x \\
& =(p-1)\left(\frac{\Lambda_{m}(n-1)}{\beta_{m}(n-1)}\right)^{-1 /(p-1)} .
\end{aligned}
$$

Hence, we have

$$
B_{m}^{p}(n)<(p-1)^{p-1} R_{m}(n-1)\left(\frac{\Lambda_{m}(n-1)}{\beta_{m}(n-1)}\right)^{-1}
$$

where $R_{m}(n-1)=\sum_{i=n}^{\infty} \lambda_{m}(i) B_{m-1}^{p}(i)$.

Also

$$
\begin{aligned}
& -p \lambda_{m}(n) B_{m-1}(n) B_{m}^{p-1}(n) \\
= & -p \lambda_{m}(n) \beta_{m}(n) B_{m-1}(n) \frac{B_{m}^{p-1}(n)}{\beta_{m}(n)} \\
= & -p \Lambda_{m}(n)\left(B_{m}(n)-B_{m}(n+1)\right)\left(\frac{B_{m}^{p-1}(n)}{\beta_{m}(n)}\right) \\
= & -p\left(\frac{\Lambda_{m}(n)}{\beta_{m}(n)}\right) B_{m}^{p}(n)+P\left(\frac{\Lambda_{m}(n)}{\beta_{m}(n)}\right) B_{m}(n+1) B_{m}^{p-1}(n) \\
\leq & -p\left(\frac{\Lambda_{m}(n)}{\beta_{m}(n)}\right) B_{m}^{p}(n)+\left[\frac{\Lambda_{m}(n)}{\beta_{m}(n)}\right]\left[B_{m}^{p}(n+1)+(p-1) B_{m}^{p}(n)\right] \\
= & \left(\frac{\Lambda_{m}(n)}{\beta_{m}(n)}\right)\left[B_{m}^{p}(n+1)-B_{m}^{p}(n)\right] .
\end{aligned}
$$

Thus

$$
\begin{aligned}
& \lambda_{m}(n) B_{m}^{p}(n)-\frac{\left(\beta_{m}(n)-\beta_{m}(n-1) \Lambda_{m}(n-1)\right.}{\beta_{m}(n) \beta_{m}(n-1) \lambda_{m}(n)} \lambda_{m}(n) B_{m}^{p}(n) \\
& -p \lambda_{m}(n) B_{m-1}(n) B_{m}^{p-1}(n) \\
\leq & \lambda_{m}(n) B_{m}^{p}(n)-\left(\frac{\Lambda_{m}(n-1)}{\beta_{m}(n-1)}\right) B_{m}^{p}(n)+\left(\frac{\Lambda_{m}(n-1)}{\beta_{m}(n)}\right) B_{m}^{p}(n) \\
& +\left(\frac{\Lambda_{m}(n)}{\beta_{m}(n)}\right) B_{m}^{p}(n+1)-\left(\frac{\Lambda_{m}(n)}{\beta_{m}(n)}\right) B_{m}^{p}(n) \\
= & \left(\frac{\Lambda_{m}(n)}{\beta_{m}(n)}\right) B_{m}^{p}(n+1)-\left(\frac{\Lambda_{m}(n-1)}{\beta_{m}(n-1)}\right) B_{m}^{p}(n)
\end{aligned}
$$

for $n=1,2,3, \ldots$, .

By adding the above inequalities for $n=1,2,3, \ldots, N$ and using (11), we have

$$
\sum_{n=1}^{N} \lambda_{m}(n) B_{m}^{n}(n)-\sum_{n=1}^{N} \frac{\left(\beta_{m}(n)-\beta_{m}(n-1)\right) \Lambda_{m}(n-1)}{\beta_{m}(n) \beta_{m}(n-1) \lambda_{m}(n)} \lambda_{m}(n) B_{m}^{p}(n)
$$




$$
\begin{aligned}
& -p \sum_{n=1}^{N} \lambda_{m}(n) B_{m-1}(n) B_{m}^{p-1}(n) \\
\leq & \left(\frac{\Lambda_{m}(N)}{\beta_{m}(N)}\right) B_{m}^{p}(N+1) \\
< & (p-1)^{p-1} R_{m}(N) .
\end{aligned}
$$

Using (12) and the assumption that $k_{m} \leq p$, we obtain

$$
\left.\sum_{n=1}^{N} \lambda_{m}(n) B_{m}^{p}(n)<K_{m}\left(\sum_{n=1}^{N} \lambda_{m}(n) B_{m-1}(n)\right) B_{m}^{p-1}(n)+\varepsilon_{m}(N)\right)
$$

where $\varepsilon_{m}(N)=\frac{1}{p}(p-1)^{p-1} R_{m}(N)$ tend to zero when $N \rightarrow \infty$.

Applying Hölder's inequality to the right hand side of (15) gives

$$
\left(\sum_{n=1}^{N} \lambda_{m}(n) B_{m-1}(n) B_{m}^{p-1}(n)\right) \leq\left(\sum_{n=1}^{N} \lambda_{m}(n) B_{m-1}^{p}(n)\right)^{1 / p}\left(\sum_{n=1}^{N} \lambda_{m}(n) B_{m}^{p}(n)\right)^{(p-1) / p}
$$

so that

$$
\sum_{n=1}^{N} \lambda_{n}(n) B_{m}^{p}(n)<k_{m}\left[\left(\sum_{n=1}^{N} \lambda_{m}(n) B_{m-1}^{p}(n)\right)^{1 / p}\left(\sum_{n=1}^{N} \lambda_{m}(n) B_{m}^{p}(n)\right)^{(p-1) / p}+\varepsilon_{m}(N)\right] .
$$

By (16), we have

$$
\begin{aligned}
\left(\sum_{n=1}^{N} \lambda_{m}(n) B_{m}^{p}(n)\right)^{1 / p}< & k_{m}\left[\left(\sum_{n=1}^{N} \lambda_{m}(n) B_{m-1}^{p}(n)\right)^{1 / p}\right. \\
& \left.+\varepsilon_{m}(N) /\left(\sum_{n=1}^{N} \lambda_{m}(n) B_{m}^{p}(n)\right)^{(p-1) / p}\right] \ldots
\end{aligned}
$$

By letting $N$ tend to infinity in (17) and raising the result to the pth power, we have

$$
\sum_{n=1}^{\infty} \lambda_{m}(n) B_{m}^{p}(n) \leq k_{m}^{p} \sum_{n=1}^{\infty} \lambda_{m}(n) B_{m-1}^{p}(n)
$$

for $m=1,2, \ldots, M$. Now, since $\lambda_{1}(n) \geq \lambda_{2}(n) \geq \cdots \geq \lambda_{M}(n)$ for $n=1,2,3, \ldots$, we have

$$
\sum_{n=1}^{\infty} \lambda_{M}(n) B_{M}^{p}(n) \leq\left(\pi_{m=1}^{M} k_{m}\right)^{p} \sum_{n=1}^{\infty} \lambda_{1}(n) B_{0}^{p}(n) .
$$

This is the desired inequality (13).

Remark 4 . We note that Theorem 4 is a discrete analogue of Theorem 4 in [7] when $M=1$. Also we note that the inequality (2) is the special case of the inequality (13) 
when $M=1, k_{1}=p$ and $\beta_{1}(n)=1$, for $n=1,2,3, \ldots$, which shows the constant in (13) is the best possible.

The following Theorems are discrete analogue results given by Pachpatte in [10] which claim thier origin in the Copson's inequality givin in (1) and (2).

Theorem 5. Let $H$ be defined as in Theorem 2 and for $n=1,2,3, \ldots$, and $j=1,2$, let

$$
\begin{aligned}
& p_{j}>1,1 / p_{1}+1 / p_{2}=1, \lambda(n)>0, \beta_{j}(n)>0, a_{j}(n)>0, \\
& \Lambda_{j}(n)=\sum_{i=1}^{n} \lambda(i) \beta_{j}(i), A_{j}(n)=\sum_{i=1}^{n} \lambda(i) \beta_{j}(i) a_{j}(i) \text { and } \sum_{n=1}^{\infty} \lambda(n) H^{p_{j}}\left(a_{j}(n)\right)
\end{aligned}
$$

converges. If there exists $U_{j_{k}} \geq p_{j} /\left(p_{j}-1\right)$ such that

$$
\begin{gathered}
p_{j}-1+\frac{\left(\beta_{j}(n+1)-\beta_{j}(n)\right)-\Lambda_{j}(n)}{\beta_{j}(n+1) \beta_{j}(n) \lambda(n)} \geq \frac{p_{j}}{U_{j}}, \text { then } \\
\sum_{n=1}^{\infty} \lambda(n) H\left(\frac{A_{1}(n)}{\Lambda_{1}(n)}\right) H\left(\frac{A_{2}(n)}{\Lambda_{2}(n)}\right) \leq \frac{U_{1}^{p_{1}}}{p_{1}} \sum_{n=1}^{\infty} \lambda(n) H^{p_{1}}\left(a_{1}(n)\right)+\frac{U_{2}^{p_{2}}}{p_{2}} \sum_{n=1}^{\infty} \lambda(n) H^{p_{2}}\left(a_{2}(n)\right) .
\end{gathered}
$$

Proof. By the elementary inequality (see $[9$, p.30])

$$
x y \leq \frac{1}{p_{1}} x^{p_{1}}+\frac{1}{p_{2}} y^{p_{2}}
$$

where $x, y \geq 0, p_{1}>1$ and $1 / p_{1}+1 / p_{2}=1$, we observe that

$$
\sum_{n=1}^{\infty} \lambda(n) H\left(\frac{A_{1}(n)}{\Lambda_{1}(n)}\right) H\left(\frac{A_{2}(n)}{\Lambda_{2}(n)}\right) \leq \frac{1}{p_{1}} \sum_{n=1}^{\infty} \lambda(n) H^{p_{1}}\left(\frac{A_{1}(n)}{\Lambda_{1}(n)}\right)+\frac{1}{p_{2}} \sum_{n=1}^{\infty} \lambda(n) H^{p_{2}}\left(\frac{A_{2}(n)}{\Lambda_{2}(n)}\right) .
$$

Now a suitable application of Theorem 2 when $M=1$ on the right side of the above inequality yields the required inequality in (19) and the proof of Theorem 5 is complete.

Theorem 6. For $n=1,2,3, \cdots$, and $j=1,2$, let $p_{j}, a_{j}(n), \Lambda_{j}(n)$ be defined as in theorem 5 and let $\lambda(n-1), \Lambda_{j}(0)=0,<\beta_{j}(n-1)>$, be non-increasing positive sequence and $B_{j}(n)=\sum_{i=n}^{\infty} \frac{\lambda(i) B_{j}(i) a_{j}(i)}{\Lambda_{j}(i)}$, and further let $\sum_{n=1}^{\infty} \lambda(n) a_{j}^{p_{j}}(n)$ converge. If there exists $V_{j} \geq P_{j}$ such that

$$
1-\frac{\left(\beta_{j}(n)-\beta_{j}(n-1)\right) \Lambda_{j}(n-1)}{\beta_{j}(n) \beta_{j}(n-1) \lambda(n)} \geq \frac{P_{j}}{V_{j}}
$$

then

$$
\sum_{n=1}^{\infty} \lambda(n) B_{1}(n) B_{2}(n) \leq \frac{V_{1}^{p_{1}}}{p_{1}} \sum_{n=1}^{\infty} \lambda(n) a_{1}^{p_{1}}(n)+\frac{V_{2}^{p_{2}}}{p_{2}} \sum_{n=1}^{\infty} \lambda(n) a_{2}^{p_{2}}(n) .
$$


Proof. By the same steps as in the proof of Theorem 5 with suitable modifications and replacing Theorem 2 by Theorem 4.

Remark 5. If we take $H(u)=u, p_{1}=p_{2}=2, a_{j}(n)=a(n), \Lambda_{j}(n)=\Lambda(n)$ and $A_{j}(n)=A(n) ; B_{j}(n)=B(n)$ in Theorem 5 and Theorem 6 , then Theorem 5 and Theorem 6 reduce respectively to

$$
\sum_{n=1}^{\infty} \lambda(n)\left(\frac{A(n)}{\Lambda(n)}\right)^{2} \leq\left(\frac{U_{1}^{2}+U_{2}^{2}}{2}\right) \sum_{n=1}^{\infty} \lambda(n) a^{2}(n)
$$

and

$$
\sum_{n=1}^{\infty} \lambda(n) B^{2}(n)^{\cdot} \leq\left(\frac{V_{1}^{2}+V_{2}^{2}}{2}\right) \sum_{n=1}^{\infty} \lambda(n) a^{2}(n) .
$$

We note that the inequalities obtained in (20) and (21) are the variants of Copson's inequality givin in (1) and (2).

Theorem 7. Let $H, \lambda(n), \beta_{j}(n), a_{j}(n), \Lambda_{j}(n), A_{j}(n)$ be defined as in Theorem 5 for $j=1,2$ and let $p_{1}, p_{2} \geq 1, \sum_{n=1}^{\infty} \lambda(n) H^{p_{1}+p_{2}}\left(a_{j}(n)\right)$ converge. If there exists $S_{j} \geq$ $\left(p_{1}+p_{2}\right) /\left(p_{1}+p_{2}-1\right)$, such that

$$
\left(p_{1}+p_{2}\right)-1+\frac{\left(\beta_{j}(n+1)-\beta_{j}(n)\right) \Lambda_{j}(n)}{\beta_{j}(n+1) \beta_{j}(n) \lambda(n)} \geq \frac{p_{1}+p_{2}}{S_{j}}, \text { for } j=1,2,
$$

then

$$
\begin{aligned}
\sum_{n=1}^{\infty} \lambda(n) H^{p_{1}}\left(\frac{A_{1}(n)}{\Lambda_{1}(n)}\right) H^{p_{2}}\left(\frac{A_{2}(n)}{\Lambda_{2}(n)}\right) & \\
\leq & \left(\frac{p_{1}}{p_{1}+p_{2}}\right) S_{1}^{\left(p_{1}+p_{2}\right)} \sum_{n=1}^{\infty} \lambda(n) H^{\left(p_{1}+p_{2}\right)}\left(a_{1}(n)\right) \\
& \quad+\left(\frac{p_{2}}{p_{1}+p_{2}}\right) S_{2}^{\left(p_{1}+p_{2}\right)} \sum_{n=1}^{\infty} \lambda(n) H^{\left(p_{1}+p_{2}\right)}\left(a_{2}(n)\right)
\end{aligned}
$$

Proof. By the elementary inequality (see[4])

$$
p_{1} x^{p_{1}+p_{2}}+p_{2} y^{p_{1}+p_{2}}-\left(p_{1}+p_{2}\right) x^{p_{1}} y^{p_{2}} \geq 0
$$

where $x, y \geq 0$ and $p_{1}, p_{2}>0$ are real, we observe that

$$
\begin{aligned}
\sum_{n=1}^{\infty} \lambda(n) H^{p_{1}}\left(\frac{A_{1}(n)}{\Lambda_{1}(n)}\right) H^{p_{2}}\left(\frac{A_{2}(n)}{\Lambda_{2}(n)}\right) \leq & \left(\frac{p_{1}}{p_{1}+p_{2}}\right) \sum_{n=1}^{\infty} \lambda(n) H^{\left(p_{1}+p_{2}\right)}\left(\frac{A_{1}(n)}{\Lambda_{1}(n)}\right) \\
& +\left(\frac{p_{2}}{p_{1}+p_{2}}\right) \sum_{n=1}^{\infty} \lambda(n) H^{\left(p_{1}+p-2\right)}\left(\frac{A_{2}(n)}{\Lambda_{2}(n)}\right)
\end{aligned}
$$


Now a suitable application of Theorem 2 when $M=1$ on the right side of the above inequality yields the required inequality in (22) and the proof of Theorem 7 is complete.

Theorem 8. Let $\lambda(n), \beta_{j}(n), a_{j}(n), \Lambda_{j}(n), B_{j}(n)$ be defined as in Theorem 6 and let $p_{1}, p_{2} \geq 1, \sum_{n=1}^{\infty} \lambda(n) a_{j}^{p_{1}+p_{2}}(n)$ converge. If there exists $T_{j} \geq p_{1}+p_{2}$ such that

$$
1-\frac{\left(\beta_{j}(n)-\beta_{j}(n-1)\right) \Lambda_{j}(n-1)}{\beta_{j}(n) \beta_{j}(n-1) \lambda(n)} \geq \frac{p_{1}+p_{2}}{T_{j}} \quad \text { for } j=1,2
$$

then

$$
\begin{aligned}
\sum_{n=1}^{\infty} \lambda(n) B_{1}^{p_{1}}(n) B_{2}^{p_{2}}(n) \leq & \left(\frac{p_{1}}{p_{1}+p_{2}}\right) T_{1}^{\left(p_{1}+p_{2}\right)} \sum_{n=1}^{\infty} \lambda(n) a_{1}^{p_{1}+p_{2}}(n) \\
& +\left(\frac{p_{2}}{p_{1}+p_{2}}\right) T_{2}^{\left(p_{1}+p_{2}\right)} \sum_{n=1}^{\infty} \lambda(n) a_{2}^{p_{1}+p_{2}}(n)
\end{aligned}
$$

Proof. By the same steps as in the proof of Theorem 7 with suitable modifications and replacing Theorem 2 by Theorem 4 .

Remark 6. Theorem 2 in [12] is the special case of the Theorem 7 when $\beta_{j}(n)=1$, $S_{j}=\left(p_{1}+p_{2}\right) /\left(p_{1}+p_{2}-1\right)$, for $j=1,2$, and if we take $H(u)=u, p_{1}=p_{2}=p$, $a_{j}(n)=a(n), \Lambda_{j}(n)=\Lambda(n), A_{j}(n)=A(n) ; B_{j}(n)=B(n)$ in Theorem 7 and Theorem 8 , then Theorem 7 and Theorem 8 reduce respectively to

$$
\sum_{n=1}^{\infty} \lambda(n)\left(\frac{A(n)}{\Lambda(n)}\right)^{2 p} \leq\left(\frac{S_{1}^{2 p}+S_{2}^{2 p}}{2}\right) \sum_{n=1}^{\infty} \lambda(n) a^{2 p}(n)
$$

and

$$
\sum_{n=1}^{\infty} \lambda(n) B^{2 p}(n) \leq\left(\frac{T_{1}^{2 p}+T_{2}^{2 p}}{2}\right) \sum_{n=1}^{\infty} \lambda(n) a^{2 p}(n) .
$$

We note that the inequalities obtain in (23) and (24) are variants of copson's inequality given in (1) and (2).

\section{References}

[1] E. T. Copson, "Note on a series of positive terms," J. London Math. Soc., 2(1927), 9-12.

[2] E. T. Copson, "Note on series of posistive terms," J. London Math. Soc., 3(1928), 49-51.

[3] G. S. Davies and G. M. Petersen, "On an inequality of Hardy's (II)," Quart. J. Math. Oxford, 15(2)(1964), 35-40.

[4] G. H. Hardy, J. E. Littlewood, and G. Polya, Inequalities, Cambridge Univ, Press, Cambridge, 1952. 
[5] D. Y. Hwang and G. S. Yang, "Note on discrete Hardy's inequality," Tumkang J. Math., 21(4)(1990), 333-336.

[6] P. D. Johnson, Jr. and R. N. Mohapatra, "Inequalities involving lower triangular matrices," Proc. London Math. Soc., 41(3)(1980). 83-137.

[7] N. Levinson, "Generalizations of an inequalities of Hardy," Duke Math. J., 31(1964), 389394.

[8] E. R. Love, "Generalizations of an inequalities of hardy," J. London Math. Soc., 30(2) (1984), 431-440.

[9] D. S. Mitrinovic, Analytic inequalities, Springer-Verlag, Berlin New York, 1970.

[10] B. G. Pachpatte, "On some integral inequalities Similar to Hardy's inequality," J. Math. Anal. Apṕl., 129(1988), 596-606.

[11] B. G. Pachpatte and E. R. Love, "On some new inequalities related to Hardy's integral inequality," J. Math. Anal. Appl., 149(1990), 17-25.

[12] B. G. Pachpatte, "A note on copson's inequality involving series of positive term," Tamkang J. Math.; 21(1)(1990), 13:19.

Department of Electrical Engineering, Kuang Wu Institute, Taipei, Taiwan R. O. C. 